\title{
DETERMINAÇÃO DO PERFIL ELETROCARDIOGRÁFICO EM RATOS, UTILIZANDO TÉCNICA NÃO INVASIVA, PROVENIENTES DO BIOTÉRIO DAS FACULDADES NOVA ESPERANÇA-PB
}

\author{
João Vinícius Barbosa Roberto' \\ Michael Sarmento Furtado " \\ Joyce Hellen Nascimento Paulino" \\ Vladimir Lenin de Sousa A. Araújo" \\ Bruno Araújo Novais Lima III \\ Ivson Cartaxo BragaIV
}

\begin{abstract}
RESUMO
Os estudos baseados em experimentação animal têm exercido grande relevância na efetivação das pesquisas e para a Ciência, tornando-se um modelo pelo qual é possível inferir comparações biológicas com o organismo humano saudável ou diante de uma situação patológica, tendo em vista os princípios éticos concernentes aos trabalhos científicos que envolvem animais. Nesse contexto, pesquisadores na área cardiovascular têm utilizado essa ferramenta para estudar e compreender os agravos que se estabelecem no coração e levam a sua disfunção fisiológica modificando, entre outros aspectos, o sistema de condução elétrica deste órgão, responsável por aferir a saúde macroscópica do miocárdio, como também a frequência, força e ritmo dos batimentos. Para monitorar a atividade elétrica cardíaca utiliza-se preferencialmente o método do Eletrocardiograma, por ser um exame de fácil manuseio, baixo custo e eficaz em seus resultados. Portanto, baseados nas diretrizes dos princípios éticos e relevância do estudo do monitoramento cardíaco, buscou-se descrever e determinar o padrão eletrocardiográfico de ratos Wistar provenientes do Biotério João Bezerra de Lima, das Faculdades Nova Esperança- PB, utilizando uma metodologia não-invasiva. Foram utilizados 30 ratos machos, jovens-adultos, da linhagem wistar, pesando entre 200-300 gramas que, após sedados, foram posicionados e conectados ao eletrocardiógrafo para registro da atividade cardíaca. Os valores encontrados se assemelham ao da literatura, havendo divergência apenas na frequência cardíaca, intervalo R-R, QT e QRS, o qual atribui-se aos diversos estímulos e estresse submetidos pela dinâmica do exame. É de suma importância a construção de um método de seleção de animais de laboratório que defina o padrão de normalidade para que, por conseguinte, sejam feitos estudos baseados em tais perfis. Contudo, a dinâmica de manuseio dos materiais no experimento reduz a qualidade do traçado cardiográfico, mas fica evidente que é possível a realização de ECG de forma não invasiva, favorecendo o bem-estar animal e determinando padrões viáveis.
\end{abstract}

\section{PALAVRAS-CHAVE}

Coração. Eletrocardiograma. Rattus.

I. Doutor em Medicina Veterinária pela Universidade Federal de Campina Grande. Professor das Faculdades Nova Esperança - João Pessoa-PB.E-mail: profvinicius@facene.com.br.

II. Discentes do curso de Medicina das Faculdades Nova Esperança - João Pessoa-PB; aluno do Programa de Iniciação Científica - PRICEA - FACENE/FAMENE.

III. Aluno do curso de Medicina das Faculdades Nova Esperança - João Pessoa-PB.

IV. Cardiologista da Policlínica-Escola das Faculdades Nova Esperança - João Pessoa-PB. 


\section{INTRODUÇÃO}

A procura pelo aumento da expectativa de vida é infindável e desafiadora no que tange a prevenção, tratamento e cura dos inúmeros agravos à saúde. A tecnologia tem sido cada vez mais útil neste processo, porém sua característica essencialmente inorgânica a limita quanto a representação dos mecanismos do corpo humano, levando os pesquisadores à tentativa de ampliar este limite, ao associar as inúmeras vantagens da tecnologia à experimentação, utilizando animais, cujas semelhanças biológica e gênica, com o ser humano, permitem a compreensão e realização de estudos que contribuem diretamente no avanço da Comunidade Científica ${ }^{1}$.

Tal associação tem fundamental importância para a prática médica uma vez que o organismo vivo de um animal é capaz de demonstrar o comportamento químico e molecular, frente a quebra do equilíbrio homeostático, somado a um aparato técnico-científico de conhecimentos e instrumentos que identifiquem e contabilizem as causas deste desequilíbrio. A partir desses elementos, estipula-se a melhor maneira de intervir para alcançar o reestabelecimento da saúde do organismo. O resultado é, entre eventuais erros e acertos, novidades terapêuticas e inovação de procedimentos, além de descobertas acerca da complexidade do próprio organismo humano.

Os determinantes para o sucesso dos estudos são inúmeros, incluindo a escolha do modelo animal, que deve ser baseada naquele que melhor responda ao experimento, de modo que qualquer pesquisador possa ter acesso aos mesmos resultados. Os ratos wistar jovens são uma boa opção pelo tamanho reduzido, fácil manuseio, nutrição variada, adaptação ao cativeiro mas, principalmente, se tratando deste trabalho, pelo critério estritamente cardiopático, já que estes animais foram, em tese, sub- metidos a menores níveis de estresse em decorrência do tempo vivido 2,3 .

A avaliação dos parâmetros clínicos dos animais corresponde aos primeiros passos no estudo da interação molecular entre suas células, tecidos e órgãos, visando estabelecer um padrão na perspectiva de um organismo saudável e, posteriormente, o estudo dos atenuantes que levam a ruptura da homeostase e como o organismo se comporta nesta condição.

Pesquisadores da área cardiovascular têm aplicado esta avaliação no sistema circulatório dos seres vivos com o objetivo de estudar o seu principal órgão, o coração. Este, nos mamíferos, apresenta quatros câmaras conformadas em um esqueleto fibroso, composto de quatro anéis tendinosos circundando seus quatro orifícios valvulares. Sua principal função é fazer o bombeamento do sangue, mas isto só lhe é possível devido a sua capacidade de se despolarizar rapidamente originando uma corrente elétrica que se inicia no átrio direito, mais especificamente, no Nódulo Sinusal e percorre as câmaras cardíacas de modo a contraí-las em ritmo, frequência e força proporcionais à demanda sanguínea do organismo ${ }^{1,4}$.

Willen Einthoven, em 1902, idealizou um aparelho para registrar graficamente as correntes elétricas que participam deste fenômeno cardíaco, surgindo o eletrocardiógrafo e o eletrocardiograma. Desde então, os aparelhos utilizados e a metodologia de interpretação tem se modernizado e adquirido grande importância na prática médica e científica. Sendo de fácil manuseio, reprodutível e de baixo custo operacional, o estudo minucioso e análise metódica das ondas, dos intervalos e dos segmentos formam a base para a interpretação do eletrocardiograma normal, das patologias cardiocirculatórias e de condições extra cardíacas que modifiquem o traçado. Assim, é sabido que o intervalo PR mostra o tempo de condução nas 
câmaras atriais; já o intervalo QT corresponde a duração total da atividade elétrica ventricular. O intervalo QT varia inversamente com a frequência cardíaca ( $F C$ ) que deve ser corrigido em relação à $\mathrm{FC}$, gerando o QTc, usado para detecção da heterogeneidade da repolarização ventricular, sendo um marcador de arritmogênese, a qual é ocasionada por tempos de repolarização não homogêneos 5 .

A partir da análise dos resultados é possível inferir um padrão do perfil eletrocardiográfico dos ratos Wistar, o qual irá substanciar os demais estudos nessa área. O desenvolvimento de um tratamento de maior eficácia para a arritmia, por exemplo, só poderia ser realizado tendo como base um padrão de normalidade da referida amostra. Tal parâmetro necessita ser estabelecido, pois existem peculiaridades no perfil eletrocardiológico de animais a serem analisadas e compatibilizadas com as ondas humanas, a fim de garantir que o experimento seja verossímil, sendo de importância fundamental que, para tanto, os dados sejam coletados de forma a provocar ínfima perturbação ao animal em experimentação3.

Na prática científica, além dos componentes de conhecimento tecnológico e teórico que envolvem a pesquisa, há regulamentações e orientações que demonstram preocupação com a boa ciência, estabelecendo diretrizes e cuidados

\section{MATERIAIS E MÉTODOS}

O experimento foi conduzido no Biotério João Bezerra de Lima, pertencente as Faculdades Nova Esperança-PB. Foram utilizados 30 ratos machos, jovens-adultos, da linhagem Wistar, pesando entre 200-300 gramas. Os animais, produzidos no próprio Biotério, foram mantidos e acondicionados em caixas de polipropileno forradas com maravalha de madeira na formação da cama, contendo no máximo 4 animais por caixa, alimentados com ração e água ad libitum e especiais com os seres vivos. Essas regulamentações envolvem grande conteúdo ético no trabalho com animais de laboratório o que culmina com o conflito entre as justificativas para o uso de animais em benefício de si próprios e do homem, contrapondo ao ato de não causar dor e sofrimento a esses, sendo necessário haver o equilíbrio dos valores opostos. São considerados como legitimamente éticos os experimentos em animais que sejam de benefício direto para a vida e para a saúde humana e animal. Assim como, aqueles que não produzam benefícios diretos, mas que procuram novo saber que contribua significativamente para o conhecimento da estrutura, função e comportamento dos seres vivos. O princípio ético de reverência pela vida exige que se obtenha um 'ganho' maior de conhecimento com um 'custo' menor no número de animais utilizados e com o sofrimento dos mesmos atenuado, sendo este o princípio norteador da metodologia não invasiva, em substituição da técnica invasiva, pois traz mais vantagens para a pesquisa científica pela rapidez do processo, manejo e bem estar animal ${ }^{1,2}$.

A proposta deste trabalho foi, portanto, ao basear-se nas diretrizes dos princípios éticos e relevância do estudo do monitoramento cardíaco, descrever e determinar o padrão eletrocardiográfico de ratos Wistar, utilizando uma metodologia não-invasiva.

mantidos sob um ciclo foto periódico de $12 \mathrm{~h}$ claro e $12 \mathrm{~h}$ escuro e cuja temperatura ambiente e umidade relativa do ar são controladas e apresentaram valores de $23^{\circ} \mathrm{C} \pm 22^{\circ} \mathrm{C}$ e $40 \% \pm 10 \%$, respectivamente. A alimentação foi constituída de ração balanceada padronizada para roedores. O Estudo foi avaliado pelo CEUA FACENE/FAMENE e julgado aprovado, sob o protocolo $n^{\circ}$ 0052.2017.1.

Os animais foram pesados e, após obtido o peso, todos os animais foram se- 
dados com pentobarbital sódico, na dose de $40 \mathrm{mg} / \mathrm{kg}$ intraperitoneal e demarcados na cauda para identificação. Após sedação, os animais foram posicionados e fixados em mesa de procedimentos com auxílio de esparadrapo, em decúbito dorsal. Seguiu-se com tricotomia das faces mediais e proximais de cada membro e colocação dos eletrodos nesses locais, diretamente fixados na pele do animal. Para captação do sinal cardíaco os eletrodos foram conectados ao eletrocardiógrafo e registradas três derivações bipolares (DI, DII e DIII) e nas três derivações amplificadas (aVR, aVL e aVF) com sensibilidade $\mathrm{N}$ e velocidade de $50 \mathrm{~mm} / \mathrm{s}$ (figura 1).

Após registro da atividade elétrica cardíaca pelo eletrocardiógrafo, foi emitido o resultado para o especialista e feita a análise do traçado. Estudo feito através da análise descritiva dos dados, com a exposição dos resultados em dados numéricos e observação do padrão clinico e comportamental a respeito do processo de aferição eletrocardiográfica não invasiva.

\section{RESULTADOS E DISCUSSÃO}

As pesquisas com animais de experimentação são de grande valor para o avanço da ciência, principalmente no que se trata do desenvolvimento de fármacos, vacinas e técnicas cirúrgicas para a humanidade. A precisão atrelada aos experimentos acosta-se no fato de que cada espécie possui suas particularidades fisiológicas e que independente da espécie o bem-estar dos animais deve ser garantido ${ }^{1}$.

O eletrocardiograma é um instrumento importante e efetivo na detecção de alterações patológicas na homeostasia elétrica da musculatura cardíaca e é largamente utilizado, tanto na clínica médica, quanto na experimentação básica, além de ser de fácil domínio e interpretação. É importante frisar que os componentes do ECG em ratos normais assemelham-se aos registros obtidos nos seres-humanos, com algumas exceções, por exemplo, a onda T que nor-
Figura 1 - Animal experimental em decúbito dorsal, sedado e com eletrodos conectados nos pontos de fixação

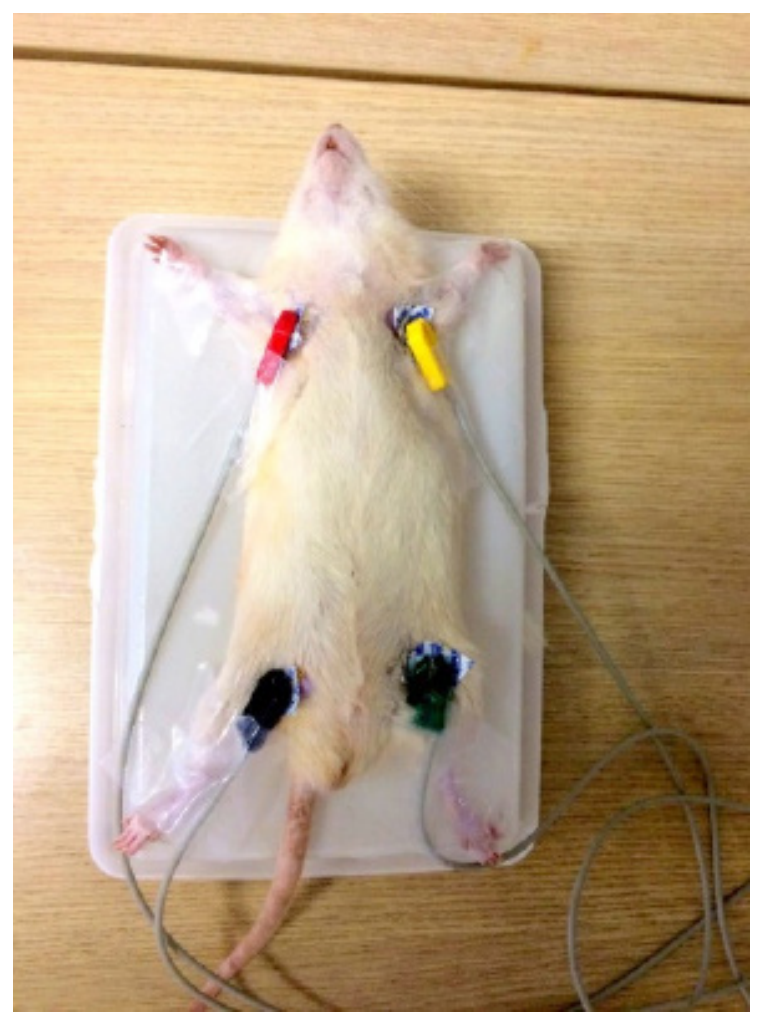

malmente é positiva e acompanha em direção contínua a onda $\mathrm{S}^{1}$.

Com relação aos registros eletrocardiográficos e suas variações em ratos, devese considerar que a estabilidade ocorre somente quando os animais atingem a vida adulta. Se considerarmos dividir a atividade eletrocardiográfica em três momentos, teremos que a primeira fase ocorre nos momentos iniciais da vida, a qual advém do início da maturação funcional e orgânica e proporciona a obtenção de níveis elevados de Frequência Cardíaca ( $F C$ ), na segunda fase ocorre diminuição/manutenção de um platô, em que FC se estabiliza. A terceira e última fase relaciona-se ao envelhecimento. Nesta, o sistema cardíaco encontra-se com atividade reduzida e, portanto, obtemos FC diminuída 6 .

No tocante à anatomia, o coração do animal em estudo ocupa a direção ân- 
tero-posterior e da direita para a esquerda, com orientação do vetor médio do eixo elétrico do QRS no plano frontal, variando de 30 a 60 graus. O peso corporal é outro parâmetro que pode interferir na orientação espacial do complexo QRS, tanto no plano horizontal, como no plano frontal ${ }^{1}$.

Na tabela 1, observam-se os valores dos parâmetros eletrocardiográficos e a frequência cardíaca de ratos Wistar não tratados.

Tabela 1 - Parâmetros eletrocardiográficos e frequência cardíaca de ratos Wistar não tratados, provenientes do Biotério João Bezerra de Lima, das Faculdades Nova Esperança-PB.

\begin{tabular}{|c|c|c|c|c|c|c|}
\hline RATO & $\begin{array}{c}\text { Intervalo } \\
\mathrm{R}-\mathrm{R}(\mathrm{S})\end{array}$ & QRS(ms) & $\begin{array}{c}\text { intervalo } \\
\text { PR(ms) }\end{array}$ & $\mathrm{QT}(\mathrm{ms})$ & QTc(ms) & FC \\
\hline 1 & 0,135 & 30 & 40 & 80 & 218 & 444 \\
\hline 2 & 0,14 & 30 & 50 & 90 & 241 & 429 \\
\hline 3 & 0,17 & 30 & 50 & 80 & 194 & 353 \\
\hline 4 & 0,15 & 40 & 40 & 90 & 232 & 400 \\
\hline 5 & 0,155 & 40 & 40 & 90 & 229 & 387 \\
\hline 6 & 0,13 & 50 & 40 & 80 & 222 & 462 \\
\hline 7 & 0,13 & 42 & 30 & 92 & 255 & 462 \\
\hline 8 & 0,124 & 50 & 40 & 90 & 256 & 484 \\
\hline 9 & 0,12 & 40 & 40 & 80 & 231 & 500 \\
\hline 10 & 0,12 & 40 & 40 & 90 & 260 & 500 \\
\hline 11 & 0,14 & 40 & 50 & 90 & 241 & 429 \\
\hline 12 & 0,13 & 30 & 40 & 80 & 222 & 462 \\
\hline 13 & 0,14 & 50 & 40 & 90 & 241 & 429 \\
\hline 14 & 0,14 & 40 & 40 & 100 & 267 & 429 \\
\hline 15 & 0,14 & 30 & 40 & 80 & 214 & 429 \\
\hline 16 & 0,14 & 40 & 40 & 80 & 214 & 429 \\
\hline 17 & 0,14 & 40 & 40 & 80 & 214 & 429 \\
\hline 18 & 0,12 & 40 & 30 & 80 & 256 & 484 \\
\hline 19 & 0,14 & 30 & 40 & 80 & 222 & 429 \\
\hline 20 & 0,14 & 50 & 40 & 100 & 218 & 500 \\
\hline 21 & 0,12 & 40 & 40 & 92 & 194 & 462 \\
\hline 22 & 0,14 & 30 & 40 & 90 & 241 & 429 \\
\hline 23 & 0,13 & 40 & 40 & 80 & 241 & 429 \\
\hline 24 & 0,13 & 40 & 40 & 90 & 267 & 500 \\
\hline 25 & 0,14 & 30 & 50 & 90 & 214 & 429 \\
\hline 26 & 0,14 & 30 & 40 & 80 & 214 & 429 \\
\hline 27 & 0,12 & 50 & 40 & 80 & 222 & 400 \\
\hline 28 & 0,14 & 30 & 40 & 80 & 256 & 462 \\
\hline 29 & 0,14 & 50 & 50 & 90 & 255 & 353 \\
\hline 30 & 0,14 & 50 & 50 & 80 & 229 & 387 \\
\hline
\end{tabular}

Eletrocardiograma: derivação II; velocidade $50 \mathrm{~mm} / \mathrm{s}$ 
O intervalo PR pode ser descrito como a medida desde o início da onda $\mathrm{P}$ até o início do QRS, o qual demonstra o tempo decorrido pelo estímulo, até a despolarização ventricular, após passar pelo feixe de Hiss e fibras de Purkinje. Os resultados mostraram uma média de 41,17 para esse intervalo PR. Valores esses obtidos que não divergem muito dos verificados por outros autores, como $37,8^{6}$ e em relação ao intervalo $R-R$, o qual representa a distância entre duas ondas $R$ mostrando um batimento. Os resultados mostraram valor médio de 0,13 com variação mínima entre o grupo estudado, mas com valores bem abaixo do obtido em outros estudos, como $0,5^{6}$. Acredita-se que esta diferença deu-se sobre a dinâmica da coleta de dados e os diversos estímulos sofridos pelos animais na coleta de dados.

A dispersão do intervalo QTc é uma medida do intervalo QT em que se considera a FC. Nessa situação, o intervalo QTc varia inversamente em relação à FC. Foi usada a fórmula de Bazzet para o cálculo do intervalo QTC, sendo a operação realizada da seguinte forma: intervalo QT medido dividido pela raiz quadrada do intervalo entre duas ondas $R$ sucessivas ( $R-R$ ), no mesmo batimento em que se mediu o QT3. Os resultados mostraram que a média do intervalos de QTC foi de 232,4. Outros estudos demonstraram resultados parecidos, encontraram valores médios para os intervalos de $212,26^{6,7}$.

\section{CONSIDERAÇÕES FINAIS}

Destaca-se, portanto, a importância do uso de eletrocardiograma para seleção de animais em qualquer que seja o procedimento de pesquisa, já que retrata fielmente anomalias na musculatura cardíaca que podem ser detectadas em repouso. São elas, variações elétricas que acompanham o aumento da idade, condições isquêmicas do miocárdio, doenças de fundo neurológico, alterações
Em relação ao intervalo QT, parâmetro eletrocardiográfico que considera a duração de uma sístole elétrica, medindo a quantidade de tempo requerida para a despolarização e repolarização ventricular, apesar de acompanhar a dinâmica funcional cardíaca descrita em humanos. Tal qual referido na literatura ${ }^{8}$, os resultados mostraram uma média de 86 para os valores obtidos no intervalo QT.

Quando comparado aos valores obtidos em outros estudos, Pezolato e colaboradores ${ }^{6}$ obtiveram uma valor médio de 103,57 . Assim como neste estudo ${ }^{6}$, a presente pesquisa também registrou dados para o intervalo PR discordantes da literatura. Isso se deu possivelmente devido a dinâmica e estímulos presentes na coleta de dados.

Em relação ao intervalo $\mathrm{QRS}$, este representa a ativação elétrica ventricular, sendo medido desde a primeira onda, seja ela $Q$ até o final da última onda (geralmente sua expressão varia de 50 a 100ms, estando esse intervalo diminuído em diversas afecções ou em indivíduos obesos).

No que diz respeito ao intervalo QRS, observa-se na tabela 1, uma média de 39,06. Valores estes abaixo dos obtidos por outros autores 6,7 .

Quanto à frequência cardíaca, dos anteriores. Neste contexto, ressalta-se a influência das condições ambientais no momento do experimento e também dos estímulos sofridos pelos animais na coleta de dados.

hidroeletrolíticas ou síndromes congênitas. Esse processo de seleção é de muita significância para as pesquisas práticas, visto que alterações indiretas no equilíbrio homeostático dos animais podem causar alterações nos resultados.

Observou-se, através da dinâmica do experimento, uma redução na qualidade do traçado do eletrocardiógrafo, devido, provavelmente, à baixa fixação do eletro- 
do na pele do animal.

A realização do ECG em ratos de forma não invasiva é possível e mais estu- dos devem ser realizados, no sentido de aprimorar esta metodologia e favorecer o bem-estar animal.

\title{
DETERMINATION OF THE ELECTROCARDIOGRAPHIC PROFILE IN RATS, USING NON-INVASIVE TECHNIQUE, FROM THE BIOTERIUM OF THE NOVA ESPERANÇA COLLEGE IN PARAÍBA STATE
}

\begin{abstract}
The research with animals has great relevance for the accomplishment of the studies in humans and for the Science, and has become a model to make comparisons between the healthy person or undergoing a pathological situation, based on the ethics of scientific animal research. However, researchers in the cardiovascular area use this tool to study and understand heart disease and physiological dysfunctions by modifying the electrical conduction system of the heart and is responsible for measuring the macroscopic health of the heart muscle, the frequency, strength and rhythm of the heart. The monitoring of cardiac electrical activity prefers to use the electrocardiogram method, an easy-to-use, low-cost, and effective examination of its results. Therefore, based on the guidelines of the ethical principles and relevance of the study of cardiac monitoring, we sought to determine the electrocardiographic pattern of Wistar rats from the João Bezerra de Lima Laboratory, in the Nova Esperança College, in Paraíba state using a non-invasive method. Were used 30 adult male wistar rats weighing between 200-300 grams, which after sedation were positioned and connected to the electrocardiograph to record heart activity. The values found are similar to those in the literature, but a difference in heart rate, R-R interval, QT and QRS, attributed to the various stimulus and stresses submitted by the exam dynamics. It is very important to construct a laboratorial selection of animals method that defines the normality standard so that studies based on such profiles are made, however, the dynamics of material handling in the experiment reduces the quality of the cardiographic tracing, but it is clear that it is possible to perform ECG in a non-invasive way, favoring the well-being of animals.
\end{abstract}

\section{KEYWORDS}

Heart. Eletrocardiogram. Rattus.

\section{REFERÊNCIAS}

1 Christofoletti DC, Pezolato VA, Abreu MFR, Mascarin AL, Silva CA. Eletrocardiografia: um método útil na seleção de animais de experimentação. Saúde Rev. 2013 Jan-Abr. 13 (33): p.39-46.

2 Andrade A, Pinto SC, Oliveira RS. Animais de Laboratório: criação e experimentação. Rio de Janeiro: Fiocruz; 2002. p.19-25.

3 Pezolato VA, Mascarin AL, Ferreira RB, Dias R, Silva CA. Monitoramento eletrocardiográfico no desenvolvimento de ratos Wistar. Arq. Bras. Med. Veterinario. Zootec. 2017, 69 (1): p.39-48.
4 Tucci PJF. Características fisiopatológicas do modelo de insuficiência cardíaca pós-infarto do miocárdio no rato. Arq Bras Cardiol 2011; 96 (5): p.420-424.

5 Pezolato VA, Mascarin AL, Paulino PC, Silva $C A$. Padrão eletrocardiográfico de ratos após recuperação nutricional. Medicina (Ribeirão Preto) 2013; 46 (3): p.8 - 281.

6 Pezolato VA, Mascarin AL, Ferreira RB, Dias $R$, Silva CA. Acompanhamento eletrocardiográfico no desenvolvimento de ratos Wistar. [Arq. Bras. Med. Vet. Zootec] São Paulo 2017. [acesso em 07 Fev. 2018]; 69(1):[9p]. 
revista de NOVA
ciêncios

da saúdele ESPERANÇA

Disponível em: http://www.scielo.br/scielo. php?pid=S0102-09352017000100039\&scri pt=sci_abstract\&tlng=pt

7 Pezolato VA, Silva CA. Determinação do padrão eletrocardiográfico de ratos jovens e adultos. $10^{\mathrm{a}}$ Mostra Acadêmica UNIMEP. $20^{\circ}$ Congresso de Iniciação Científica. [periódico da internet] São Paulo 2012. [citado 07 Fev. 2018]; [4p]. Disponível em: http://www. unimep.br/phpg/mostraacademica/anais/ 10mostra/1/288.pdf
8 Guyton AC, Hall JE. Tratado de Fisiologia Médica. $11^{\text {a }}$ ed. Rio de Janeiro, Elsevier Ed., 2006.

9 Carvalho GD, Masseno APB, Zanini MS, Zanini SF, Porfírio LC, Machado JP, Mauad H. Avaliação clínica de ratos de laboratório (Rattus novergicus linhagem Wistar): parâmetros sanitários, biológicos e fisiológicos. [periódico da internet] Minas Gerais 2009. [acesso em 07 Fev. 2018]; 56(1): [7p]. Disponível em: http://www.ceres.ufv.br/ojs/index.php/ceres/ article/view/3393/1281 